\title{
La dimensión política de los vínculos Argentina-Chile y Argentina-Venezuela en el inicio del Siglo XXI ${ }^{1}$
}

The political dimension in Argentina-Chile and ArgentinaVenezuela bilateral links in the 21st Century

\author{
María Elena Lorenzini ${ }^{2}$
}

Resumen: El objetivo del artículo es analizar comparativamente las relaciones ArgentinaChile y Argentina-Venezuela en el período 2003-2011, identificando sus principales similitudes y diferencias en la dimensión política, marco amplio en el que se asienta una relación bilateral. En nuestro estudio, es el estado de cosas, discursos y acciones de los gobiernos en la dimensión político-diplomática tales como los principios y mecanismos institucionales que estructuran el diálogo en las diversas issue areas. Asimismo, se busca identificar cuáles son los rasgos propios de cada vínculo y las características que comparten para sobre esa base, analizar si reúnen las condiciones para ser catalogados como alianzas estratégicas.

Abstract: This article presents a comparative analysis of Argentina-Chile and ArgentinaVenezuela bilateral relations in the beginning of 21st Century, identifying their main similarities and differences in the political dimension, which comprises the broad political framework which acts as a base of the relationship. In our study, its constitutes the state of things, discourses and actions of governments such as principles upon which bilateral links rest and institutional mechanisms which structure the political dialogue in different issue areas. This paper also seeks to identify the distinctive features of these ties and shared characteristics. Then, discusses if these bilateral ties have the potential to become strategic alliances, and, how much progress they have made in the process.

Palabras Clave: Política Exterior, Relaciones Bilaterales, Alianza Estratégica, Argentina, Chile, Venezuela

Keywords: Foreign Policy, Bilateral Links, Strategic Alliance, Argentina, Chile, Venezuela

\footnotetext{
${ }^{1}$ Recibido: 3/10/2016 Aprobado: 25/04/2017

2 Doctora en Relaciones Internacionales, Universidad Nacional de Rosario. Argentina. Profesora Adjunta Teoría de las Relaciones Internacionales, Facultad de Ciencia Política y Relaciones Internacionales. Universidad Nacional de Rosario. Investigadora Consejo Nacional de Investigaciones Científicas y Técnicas (CONICET). Male_lorenzini@yahoo.com.ar
} 


\section{Introducción ${ }^{3}$}

Las relaciones argentino-chilenas y argentino-venezolanas constituyen temas prioritarios de la política externa argentina (Colacrai, 2014; Robledo, 2011; Simonoff, 2013; Miranda, 2014; Bernal-Meza, 1998). El artículo se aproxima al análisis comparado de las relaciones Argentina-Chile y Argentina-Venezuela a inicios del SXXI, identificando sus principales similitudes y diferencias en la dimensión política. Luego, indaga si los rasgos de cada vínculo resultan suficientes para catalogar las relaciones como alianzas estratégicas.

Para ello, formula un conjunto de interrogantes: ¿Cuál es su punto de partida? ¿De qué manera se desarrolló el proceso de construcción de la relación bilateral? ¿De qué modo se estructuran los mismos -principios que los orientan-, mecanismos de diálogo y si funcionan regularmente? ¿Cuáles fueron los temas conflictivos que surgieron y qué herramientas se utilizaron para su solución? ¿Los rasgos que las caracterizan, son suficientes para catalogarlas como alianzas estratégicas?

Las perspectivas teóricas que orientan el trabajo se articulan desde la interdependencia (Keohane \& Nye, 1988) y el institucionalismo liberal (Keohane, 1984; Grieco, 1988). Los supuestos de la interdependencia para el armado conceptual, son: la existencia de issue areas que funcionan con cierto grado de autonomía; la desjerarquización de la agenda de la política exterior que incide sobre la fijación de prioridades -actores y temas- y la elección del canal interestatal de las relaciones -reconociendo la existencia del transgubernamental y el transnacional- (Keohane \& Nye, 1988). Se comparte el supuesto institucionalista que considera que aún en la anarquía internacional la cooperación prolongada es posible en virtud de los principios, las reglas acordadas y su cumplimiento (Keohane, 1984; Grieco, 1988). Esto supone asignar cierto valor a las instituciones pues ordenan, limitan y regulan los comportamientos tal como se utiliza al examinar las trayectorias de ambas relaciones. Los elementos teóricos seleccionados están presentes en los conceptos de política exterior y alianza estratégica, respetando el significado asignado por los enfoques a los mismos.

La política exterior es entendida como un "instrumento estatal (...) que cuenta con diversos medios, por ejemplo diplomáticos, culturales, militares (...). Entre los primeros "se destacan capacidades, como la de asociarse con otros actores, negociar diferencias, impulsar iniciativas políticas (...)" (Miranda, 2014: 59). Las cuestiones diplomáticas se relacionan con la capacidad de asociación y con el concepto de alianza estratégica pues refieren al marco político -principios, mecanismos institucionales y de diálogo- que componen el cuadro de situación junto con los discursos y acciones de los gobiernos.

El nexo entre la dimensión política de la política exterior y alianza estratégica consiste en que, la decisión de conformarla tiene lugar en ese marco político amplio pues es la política la que fija -en mayor medida- las reglas del juego. El concepto de alianza estratégica se ha utilizado frecuentemente de dos modos. Por un lado, de una forma general y en sentido laxo que entiende que las alianzas estratégicas son "(...) un tipo de relación interestatal que por diversos motivos y factores, se distingue en cuanto a consideración e importancia del resto de las relaciones bilaterales que componen el universo diplomático de un país" (Cortés \& Creus, 2009: 120).

\footnotetext{
${ }^{3}$ Una versión preliminar de este trabajo fue expuesta en la Conferencia Conjunta FLACSO-ISA, realizada en Buenos Aires, julio 2014.
} 
Por el otro, uno específico y en sentido estricto que concibe que las alianzas estratégicas comprenden integralmente el vínculo -político, económico y militar- y se construye sobre una relación densa, profunda, basada en la confianza (Colacrai \& Lorenzini, 2006; Lorenzini 2011). Cabe subrayar que la decisión de avanzar hacia la alianza estratégica demanda un grado mayor de coordinación y planificación de las partes involucradas, otorgándole al carácter estratégico un sentido predominantemente político. Esta última definición procura dotar de contenido el concepto y evita utilizarlo como vocablo de moda.

A partir de lo antedicho, la hipótesis sostiene que las relaciones argentino-chilenas conforman un vínculo de alianza estratégica en sentido estricto ya que son densas y profundas, exhiben una trayectoria de trabajo conjunto, muestran avances parciales en la coordinación de acciones -en lo bilateral y en lo regional-; mientras que las relaciones argentino-venezolanas se inscriben en una dinámica predominantemente discursiva configurando lo que Lechini (2006: 16) denomina "inconsecuente retórica" o bien una alianza estratégica en sentido laxo, pues esta relación es menos densa, sus bases no están consolidadas, sus instituciones funcionan irregularmente y existe un menor conocimiento de las partes lo que dificulta, aún más, la tarea de planificar cursos de acción conjuntos. En la relación argentino-chilena, la intención de conformar una alianza estratégica se anunció en 2000 y desde entonces forma parte de su agenda. El relanzamiento del vínculo pos crisis del gas (2004-2007) y la puesta en práctica del Tratado de Maipú, son indicios de avance en la construcción de esta alianza estratégica en sentido estricto, aunque el proceso permanece inconcluso. Mientras que la idea de conformar una alianza estratégica entre Argentina y Venezuela surge a partir de una supuesta convergencia ideológica, pero se trata de un vínculo con una trayectoria escasa en el que parecen no haberse calculado ajustadamente los recursos necesarios para implementarla, aproximándose así a la idea de una alianza estratégica en sentido laxo.

Desde lo metodológico, el análisis se elabora considerando los rasgos compartidos (Sthinchcombe, 1978) y los diferenciadores (Przeworski \& Teune, 1970). Se opta por una estrategia que mixtura elementos de ambos enfoques con el objetivo de tomar lo mejor de ellos (Collier, 1994). Se adopta el enfoque comparativo como dispositivo heurístico de los vínculos y los procesos de alianza estratégica.

Para analizar la dimensión política el artículo se inicia, explicitando el punto de partida teórico y sus conexiones con los conceptos centrales. Luego, hace un racconto sintético de los antecedentes más destacados de cada vínculo para elaborar una radiografía de ellas que comprenda los hechos y procesos acaecidos en el período. Sobre esa base de información, en la sección final, se identifican sus semejanzas y diferencias y se reflexiona acerca de si sus características hacen de ellos alianzas estratégicas.

\section{Los antecedentes más destacados}

En 1984 Argentina y Chile firmaron el Tratado de Paz y Amistad para resolver el conflicto por el Canal de Beagle. Simonoff afirma que "si bien Chile ganó la soberanía de las islas en disputa, el nuevo tratado supuso no sólo un nuevo paradigma para la resolución de los conflictos pendientes (...) sino también abrió las posibilidades de concertar una política más cooperativa" (2013: 22). Así se inició una nueva etapa en la relación 
bilateral denominada nueva política ${ }^{4}$ (Colacrai \& Lorenzini, 2006). Desde entonces, ambos Estados decidieron poner fin a un pasado signado por una imagen recíproca de rivalidad intensa ${ }^{5}$. El acuerdo celebrado entre el gobierno autoritario de Pinochet y el gobierno democrático de Alfonsín fue el punto de inicio sobre el que empezó a construirse un nuevo modo de vinculación.

En 1991 Aylwin-Menem renovaron los votos de confianza dando un nuevo salto cualitativo ${ }^{6}$ con la firma de la Declaración Presidencial Conjunta Argentino-Chilena cuyo objetivo principal era la consolidación de una frontera de paz (Colacrai, 2004; Valdés, 1999; Rojas Aravena, 2003; Durán, 2012). También se firmó la Declaración Conjunta sobre Límites que estableció los mecanismos para resolver las 24 cuestiones territoriales y el Acuerdo de Complementación Económica № 16 (ACE 16) que regularía la liberalización comercial, las inversiones, el suministro de gas natural y la explotación conjunta de zonas de fronteras.

Durante los noventa, las relaciones se desarrollaron en un clima predominantemente cooperativo (Diamint, 2004; Simonoff, 2010; Simonoff, 2013; Robledo, 2011; Aranda \& Riquelme, 2013). El diálogo político mantuvo un excelente nivel y se crearon mecanismos para institucionalizarlo -Cumbres Presidenciales, Reuniones de Ministros de Relaciones Exteriores y de Defensa $(2+2)$, Comités de Frontera, etc.-. Otros ejemplos fueron la fuerte voluntad política de los gobiernos para resolver Hielos Continentales (Colacrai, 2004); el crecimiento comercial y la inversión (PROCHILE, 2005) y la cooperación en defensa (Alvear, 2004; Diamint, 2006). En la segunda mitad de los noventa, la renovada relación resolvió todos los litigios fronterizos; estableció un plan de integración física; implementó medidas de confianza mutua (MCM); realizó ejercicios militares, participaron conjuntamente en operaciones de mantenimiento de la paz (OMP); adoptaron una metodología para la homologación y transparencia del gasto militar; ampliaron el intercambio cultural-académico y crearon un marco institucional para la cooperación. El corolario de esta excepcionalidad positiva ${ }^{7}$ fue la declaración de Argentina-Chile como aliados estratégicos el 19 de mayo de 2000 (Ministerio de Relaciones Exteriores, Comercio Internacional y Culto, 2000). Desde entonces, la consigna de la alianza estratégica ha sido una constante en las declaraciones presidenciales y en los discursos de política exterior argentinos y chilenos.

Las relaciones Argentina-Venezuela (RBV) tienen una historia más breve y reciente ya que durante los noventa el vínculo con Caracas tuvo escasa relevancia política destacándose un moderado nivel comercial y un importante flujo de capitales emitido por empresas argentinas (Kosacoff, 1999). Al respecto, Ana María Sanjuán sostiene que Chávez había intentado impulsar una mayor aproximación bilateral con Argentina duran-

${ }^{4}$ Se caracteriza por el cambio en el patrón de la vinculación que reemplaza el conflicto por la cooperación en diversas áreas de cuestiones. Por su parte, la vieja política alude a aquel modo de vinculación signado por la desconfianza mutua, la percepción de una amenaza permanente, la idea del vecino como un rival y cuyo eje central está compuesto por la defensa del territorio, de la fronteras y de la soberanía (Colacrai \& Lorenzini, 2006; Lorenzini, 2011).

${ }^{5}$ Adaptación del autor del modelo de Wendt (1999). Se la califica como intensa para marcar la diferencia con la nueva forma de vinculación que caracteriza la relación desde los ‘90s.

${ }^{6}$ Alude al reemplazo de interacciones conflictivas por interacciones cooperativas que modificaron la forma y el contenido de la vinculación.

${ }^{7}$ Taccone (2001) resalta la magnitud del cambio en el patrón de las relaciones argentino-chilenas luego de la redemocratización. El calificativo positiva se asocia a la cooperación y es útil para marcar el cambio de signo con respecto a las características de la relación en el pasado. 
te los últimos años de la década del noventa pero, fue recién "con el gobierno de Néstor Kirchner (...) que las relaciones alcanzaron su más alto nivel” (Sanjuán, 2008: 168). Dicha situación se expresó en la firma del Convenio Integral de Cooperación (2004) y dos años después en la firma del Acuerdo para el establecimiento de una alianza estratégica (2006) que oficializó el objetivo de ambos gobiernos.

A partir de 2003, los vínculos Buenos Aires-Caracas se convirtieron en una prioridad de ambas políticas exteriores y su ascenso fue intenso y veloz (Serbín \& Serbín, 2014). Éstos se profundizaron y ganaron relevancia en el contexto de la superposición de la crisis económica y financiera que Argentina estaba superando y de la emergencia de la crisis energética derivada de la reactivación productiva. Hasta 2007 experimentaron un rápido ascenso, respondiendo a la fuerte decisión política de los presidentes y a la complementariedad de intereses. Paralelamente, el valija gate ${ }^{8}$, la nacionalización de Sidor $^{9}$ y las sospechas de pagos de coimas ${ }^{10}$ para que las empresas argentinas ingresaran al mercado venezolano, impusieron un perfil más bajo durante las administraciones de Cristina Fernández (2008-2011) y Hugo Chávez respectivamente.

\section{La primera década del Siglo XXI}

\section{Argentina-Chile: La crisis del gas, la Declaración de Punta Arenas y el Tra- tado de Maipú}

La primera década del siglo XXI puso a prueba la fortaleza de este vínculo. La crisis del gas ${ }^{11}$, el caso Apablaza ${ }^{12}$, la imposición de trabas comerciales a productos manufacturados chilenos y los problemas de la aerolínea chilena LAN para operar en el Aeroparque Jorge Newbery, fueron episodios que generaron tensión entre las administraciones argentinas -Néstor Kirchner y Cristina Fernández- y chilenas -Ricardo Lagos, Michelle Bachelet y Sebastián Piñera (Ruz, 2011; Briones \& Dockendorff, 2015).

Cuando asumió Cristina Fernández, el mayor desafío consistía en relanzar estas relaciones en un escenario pos crisis del gas (Huneeus, 2007; Flisfich, 2011). En 2008 se

${ }^{8}$ Primer episodio de sospechas sobre la transparencia de la relación cuando un agente de la policía aeroportuaria argentina, identificó al empresario Antonini Wilson, que intentaba ingresar con una valija con USD 790.550 sin declarar (Clarín, 04/08/2007, La Nación, 04/08/ 2007).

${ }^{9}$ En 2008, la RBV expropió y nacionalizó empresas. Entre ellas, Siderúrgica del Orinoco (SIDOR) del grupo Techint; 3 del grupo Rocca en el mismo sector: Techint Tubo de Acero de Venezuela, Materiales Siderúrgicos y Complejo Siderúrgico de Guayana (Beltramino, 2009; Ámbito Financiero, 24/05/2009).

${ }^{10}$ En 2010 el Defensor del Pueblo argentino, denunció el pago de comisiones del 15\% en contratos de empresas argentinas que exportaban a Venezuela a través de Palmat International (Miami) y Palmat Intertrade (Panamá). Por la falta de transparencia en la gestión de contratos y por las declaraciones off de record de algunos empresarios es que se sospecha que para ingresar al negocio se pagaban coimas (La Nación, 30/04/2010; Página12, 23/06/2010).

${ }^{11}$ La crisis del gas se inició en 2004 a partir de la decisión argentina de reducir el volumen de gas exportado a Chile a raíz de la crisis energética que comenzaba a experimentar por la recuperación económica. Ver Hunneus, 2007; Tapia \& Torres, 2007; Ceppi, 2010; Domper \& Cortés, 2008; Isbell, 2006.

${ }^{12}$ El caso Apablaza se suscitó en 2010 por la negativa del gobierno argentino de conceder la extradición del ciudadano chileno Sergio Galvarino Apablaza solicitada por el gobierno de Chile. La Comisión Nacional de Refugiados en Argentina (CONARE) decidió concederle el status de refugiado. Ver: Corte Suprema de Justicia, Fallo Apablaza, 14/09/2010; Resolución CONARE, 30/09/2010; Informe CONARE, $30 / 09 / 2010$. 
cumplirían 30 años del inicio de la mediación de Juan Pablo Il y en 2009, el 25 aniversario del Tratado de 1984. Ambas fechas tenían valor simbólico y constituían una ventana de oportunidad para reforzar la relación y restablecer la confianza.

En la Declaración de Puntas Arenas, Fernández y Bachelet sostuvieron que Argentina y Chile conforman un "modelo de integración estratégica, consolidada en valores comunes, como la paz, la democracia, la promoción y defensa de los DD.HH. y la justicia social" (2008: 1). En el contexto del documento se infiere que el vínculo es caracterizado poniendo en valor los avances experimentados luego del conflicto de 1978. También anunciaron el inicio de negociaciones para un nuevo Acuerdo, complementario al de 1984, que permita "sistematizar y ordenar los mecanismos que conforman la relación bilateral, dinamizar y asegurar el proceso de integración y enfrentar con una perspectiva de futuro y de cooperación más estrecha, las próximas décadas" (Declaración de Punta Arenas, 2008: 2).

Otro punto destacado de la agenda política fue el de la coordinación y concertación de posiciones en, al menos, cuatro issue areas: cooperación antártica y sistema antártico; Reuniones de Directores Generales de Política Exterior sobre Asuntos Multilaterales; Defensa de los principios que fundamentan el Derecho Internacional de los DDHH en instancias multilaterales y cooperación consular (Declaración de Punta Arenas, 2008). Los avances del trabajo conjunto y su continuidad en el tiempo serían un paso importante para transformar en realidad, el discurso de la alianza estratégica.

La Declaración de Punta Arenas tuvo un impacto positivo sobre el vínculo, contribuyendo a retomar el dinamismo y la fluidez que lo caracterizaba antes de la crisis del gas. Asimismo, se identifican avances orientados a la construcción de la alianza estratégica en el caso de los mecanismos de concertación de posiciones señalados. Sin embargo, es el Tratado de Maipú (2009) el que provee nuevos elementos y mecanismos que, si se ponen en práctica, podrían transformar en alianza estratégica, la densa y profunda relación existente, reforzando la cooperación y la integración (Colacrai, 2014). Esta primera meta marca la orientación general para el vínculo mientras que los otros objetivos especifican los pasos a seguir, estableciendo nuevos mecanismos institucionales para concretar la alianza estratégica.

En la dimensión política, el acuerdo impulsa acciones conjuntas para renovar la orientación de los proyectos bilaterales; consolidar la cooperación, la integración y la complementariedad en las dimensiones nacional, provincial y regional fomentando la participación de organizaciones públicas, privadas y de la sociedad civil; consolidar una cultura de paz e integración sobre bases comunes; adoptar medidas para favorecer la circulación y residencia de nacionales en ambos territorios y adoptar medidas para armonizar la legislación en materia de migraciones y previsional (Tratado de Maipú, 2009).

En lo institucional, jerarquiza la Comisión Binacional de Comercio, Inversiones y Relaciones Económicas e institucionaliza la Reunión Binacional de Ministros incorporándola a los espacios de trabajo conjunto que estructuran el vínculo. Ella, se ocupará de evaluar el desarrollo del proceso de integración. En su seno, se tomarán las medidas necesarias para alcanzar los objetivos propuestos en el acuerdo de 2009 y, deberá cumplir con las directrices emanadas de las Cumbres Presidenciales (Tratado de Maipú, 2009).

El Tratado de Maipú, también, transformó las Reuniones de Coordinación y Consultas sobre Asuntos Multilaterales entre los Directores Generales de Política Exterior en el Sistema de Consultas Permanentes de Ministerios de Relaciones Exteriores cuya función principal será coordinar posiciones frente a los foros multilaterales. A partir de la infor- 
mación revisada se infiere que Argentina y Chile comenzaron a recorrer el camino de la concertación en algunas áreas de la política exterior -DDHH, Antártida, implementación del acuerdo previsional, cooperación consular. En la dimensión regional se subrayan las posiciones asumidas, conjuntamente, frente a los episodios que amenazaron la democracia en Haití (2004), Bolivia (2005) y Honduras (2009); a la tensión entre Ecuador y Colombia (2008); en el operativo de liberación de Ingrid Betancourt (2008) y en el compromiso por fortalecer y consolidar la Unión de Naciones Suramericanas (UNASUR) como espacio amplio de cooperación y diálogo político.

En lo que respecta a la Comisión Parlamentaria Conjunta ${ }^{13}$, el Tratado estableció con mayor precisión sus funciones y su relación con las demás instituciones.

Así, se observa la existencia de principios y valores compartidos por ambos gobiernos -compromiso con la democracia, respeto por los DDHH, estado de derecho, pluralismo político, solución pacífica de controversias y un denso entramado institucional- que conforman el marco político amplio sobre el que se construye la relación. Tal como se lo indicó, la existencia de una variada trama de espacios organizacionales -Cumbres Presidenciales; Reuniones 2+2; Comité Permanente de Seguridad (COMPERSEG); Comisiones y Grupos de Trabajo Binacionales; Sistema Permanente de Consultas Políticas de Alto Nivel; Comisión Parlamentaria Conjunta; Comités de Integración; Consejo Empresarial Binacional Permanente; Comisión Binacional de Cooperación Económica e Integración Física y la Reunión Binacional de Ministros- posibilitan el intercambio de información y coordinación entre las partes.

La trayectoria de las relaciones Argentina-Chile muestra que el vínculo partía de una situación de cooperación y diálogo con episodios esporádicos de tensión -crisis del gas (2004), caso Apablaza (2010), conflicto con LAN (2011). No obstante, las administraciones Bachelet-Fernández decidieron continuar profundizando la relación donde la Declaración de Punta Arenas representó el relanzamiento del vínculo y propuso la elaboración de un nuevo acuerdo que tuviera un valor equivalente al de Paz y Amistad y que proyectara la integración bilateral en el siglo XXI. Este paso, dio lugar al Tratado de Maipú en el que se reafirmó el compromiso y la voluntad de ambos gobiernos para avanzar hacia un estadío superador (Colacrai, 2014). Fernández y Piñera se mantuvieron en esa senda aunque el caso Apablaza, las trabas comerciales argentinas y el tema LAN generaron nuevos momentos de rispidez.

\section{Argentina-Venezuela: la Reunión de Cancilleres y los principios sobre los que se construye el vínculo}

El acercamiento Argentina-RBV tuvo como punto de inflexión la Reunión de Cancilleres realizada en julio de 2003 en Buenos Aires (Sanjuán, 2008; Briceño Ruiz, 2010; Roark \& Giglio, 2010). Allí, expresaron la voluntad de ampliar la relación en los planos político, económico, industrial, comercial y cooperación técnica con énfasis en los programas sociales, lucha contra la pobreza y la exclusión. A ello se sumó, la necesidad de profundizar el vínculo para consolidar el desarrollo endógeno, fortalecer la capacidad negociadora -regional e internacional- de los Estados e impulsar proyectos en sectores

\footnotetext{
${ }^{13}$ Fue creada en 1991 en cumplimiento del Tratado de 1984. Desempeñó un rol clave para la solución de las cuestiones territoriales y en el desbloqueo del Convenio de Seguridad Social (Colacrai, 2004; Colacrai, 2010).
} 
de mutuo interés: energía, agricultura y alimentos, entre otros (Declaración de Cancilleres, 2003).

La I Cumbre Presidencial se realizó en agosto de 2003 cuando los presidentes suscribieron el Manifiesto de Buenos Aires, señalando que la relación descansa sobre "los vínculos históricos y de hermandad continental (...) basados en (...) los valores de democracia y solidaridad cooperativa" (2003: 1). Kirchner y Chávez, propusieron que los vínculos se orienten a asegurar la paz y la democracia; impulsar la justicia social, combatir la pobreza y la exclusión; fomentar una integración latinoamericana y sudamericana asentada en una agenda económica y social autónoma (Briceño Monzón, 2011; Aranda, 2013). También afirmaron que es indispensable resolver el problema de la deuda externa, recuperando la solvencia económica y sostuvieron el criterio de proporcionalidad crecimiento económico y superávit fiscal- para el pago de obligaciones (Manifiesto de Buenos Aires, 2003).

En el Comunicado Conjunto del 1 de febrero de 2005 se suma la complementariedad económica como principio ordenador para que la profundización del vínculo funcione como un puente que mejore la inserción internacional y contribuya a satisfacer sus necesidades e intereses (Comunicado Conjunto, 2005a). En agosto de 2005, los presidentes se reunieron en Buenos Aires y, afirmaron que un mundo más justo, seguro, democrático y próspero sólo podría alcanzarse por medio del multilateralismo (Comunicado Conjunto, 2005b).

A partir de lo expuesto, el vínculo Argentina-RBV descansa sobre los principios de respeto a la democracia y DD.HH.; el derecho de cada Estado para elegir libremente el modelo de desarrollo y viabilizar el pago de la deuda externa; la integración regional complementaria y solidaria; respeto mutuo y reciprocidad.

\section{El Convenio Integral de Cooperación}

El 6 de abril de 2004, se firmó el Convenio Integral de Cooperación (CIC, 2004) que convoca a participar del proceso a organismos públicos, privados y otras organizaciones. El objetivo central de la cooperación en múltiples issue areas apunta a fortalecer el desarrollo económico y la integración regional.

Según el documento, la relación bilateral cuenta con 3 mecanismos institucionalizados de diálogo: Cumbres Presidenciales; Comisión Mixta (COMIX) que con la firma del acuerdo para establecer una alianza estratégica en 2006 fue reemplazada por la Comisión Binacional de Alto Nivel (COBAN) y el mecanismo de consulta permanente entre los Ministerios de Energía y Minas (RBV) y el Ministerio de Planificación Federal (Argentina).

Las Cumbres Presidenciales constituyen uno de los pilares más importantes de la relación, teniendo en cuenta que ambos regímenes políticos son presidencialistas y que la diplomacia de cumbres es un instrumento clave. El resultado de estas reuniones se plasma en Declaraciones Presidenciales y Comunicados Conjuntos donde se enuncian los principios, el plan de trabajo, los temas y actividades a desarrollar en el corto plazo y, se revisa el estado de ejecución de los proyectos. Desde 2009, estas reuniones debían realizarse trimestralmente. Sin embargo, sólo hubo una reunión presidencial en 2010 -19 y 20 de abril- y en 2011 -29 de marzo-. Estos datos evidencian que se incumplió con la frecuencia acordada y, puede ser interpretado como un gesto para suavizar la visibilidad y bajar el perfil público del vínculo luego de las sospechas de corrupción que cuestionaron su transparencia. 
La creación de la COMIX fue acordada en el CIC y, su función sería monitorear y evaluar su cumplimiento en reuniones semestrales. También definiría las prioridades de los proyectos de cooperación a implementar. Inicialmente, hubo una alta expectativa sobre los aportes que esta instancia podría realizar para darle densidad a las relaciones pero ésta nunca se reunió.

En julio de 2006, Kirchner y Chávez firmaron un acuerdo para establecer una alianza estratégica. Allí se decidió la creación de la COBAN -reemplazó a la COMIX- y debía elaborar el plan para la concreción de la alianza estratégica, tarea que aún está pendiente. Según el acuerdo, la COBAN iniciaría sus actividades en agosto de 2006 pero, la I Reunión se desarrolló el 9 de diciembre de 2009. La COBAN comenzó a funcionar con un retraso mayor a 3 años. La II Reunión se celebró el 12 y 13 de abril de 2010 y, la III, el 28 y 29 de marzo de 2011. Desde entonces no se volvió a reunir. La estructura de la COBAN está compuesta por dos Subcomisiones: Relaciones Políticas y Relaciones Económicas. Cada una está integrada por 4 capítulos y 21 grupos de trabajo.

El mecanismo de consulta permanente entre los Ministerios de Energía y Minas y el Ministerio de Planificación Federal es el canal de diálogo que más actividad registra, aún, luego de las denuncias de corrupción y de la decisión de bajar el perfil a la relación. El rol de los ministros De Vido -Argentina- y Ramírez -RBV- fue clave. En la trayectoria de la relación, ellos se desempeñaron como los interlocutores válidos en el proceso de profundización y se observa, por ejemplo, en la conducción que ejercieron en la COBAN. Otro elemento para comprender su importancia es que sobre su cartera recae el manejo de los temas energéticos, los que experimentaron un claro ascenso en las respectivas agendas. En el caso argentino, la energía se transformó en una cuestión prioritaria vis a vis el aumento sostenido de la demanda y la progresiva pérdida del autoabastecimiento. En el caso de RBV, el petróleo constituye su principal fuente de ingreso de divisas -posee las mayores reservas probadas de hidrocarburos ultrapesados en la región-. Tales características muestran la complementariedad de intereses entre Argentina y RBV sobre esta issue area.

El vínculo Miraflores-Casa Rosada descansa sobre un conjunto de principios respeto de la democracia, DD.HH.; libertad de elegir el modelo de desarrollo; cuestiones sociales; visión crítica de EEUU y del rol de los organismos multilaterales de crédito; prioridad de la integración regional, complementariedad y solidaridad, respeto mutuo y reciprocidad. Esto se visualiza en el hecho que pensaron la complementariedad como una idea guía para atender sus intereses más urgentes: la búsqueda de socios comerciales para aumentar las exportaciones; de socios políticos que apoyaran la posición argentina en el proceso de renegociación de la deuda con el FMI y el reclamo por Malvinas, y de socios energéticos que pudieran ayudarla a resolver los problemas emergentes en esta área. Argentina necesita garantizar la seguridad energética mientras que RBV necesita alcanzar la soberanía alimentaria (Ministerio del Poder Popular para la Comunicación y la Información, 2007).

Desde 2007, las sospechas de corrupción, el singular mecanismo que regulaba el comercio bilateral y las compras de energía despertaron dudas sobre la intensidad que éste tendría en el futuro. Las Cumbres Presidenciales se celebraron de manera más espaciada -no fueron trimestrales- $y$, de acuerdo con la información oficial de ambas cancillerías, la última COBAN, tuvo lugar en marzo de 2011 cuando, originalmente, la frecuencia acordada era semestral. 


\section{Resultados del análisis comparado}

Si retomamos los interrogantes iniciales, encontramos que el punto de partida constituye la primera diferencia en los vínculos argentino-chilenos y argentinovenezolanos. Los primeros partieron de una situación de conflicto condicionada por las disputas territoriales y, los segundos, lo hicieron desde la convergencia de ideas e intereses que podían satisfacer complementándose comercialmente.

En lo que respecta al lugar que ocuparon estos vínculos en la agenda externa argentina, los argentino-chilenos fueron prioritarios desde los noventa lo que ilustra su continuidad y configura lo que Colacrai (2014) denominó una política de Estado bilateral pues se mantuvieron pese a los cambios de signo político de los gobiernos, a la profunda crisis por la que atravesó Argentina en 2001 y a los episodios puntuales de conflicto. En los temas concretos donde surgieron diferencias, las partes mantuvieron su voluntad política y recurrieron a las instituciones bilaterales para zanjarlos. Los argentinovenezolanos se configuraron como prioritarios desde 2003 y no atravesaron tensiones en el período. Esta situación genera dudas acerca de las capacidades de los gobiernos y de la eficacia de sus instituciones para resolverlas. En consecuencia, los elementos empíricos resultan insuficientes y no aportan indicios claros sobre el comportamiento de ArgentinaRBV para resolver diferencias.

El proceso de de construcción del vínculo Argentina-Chile se trata de un camino planificado, pavimentado con esfuerzo, prudencia, un carácter progresivo y una impronta acumulativa. Los distintos gobiernos tomaron los recaudos para sostener en el tiempo esta relación que se fue haciendo densa y profunda -abarca diversos niveles gubernamentales y tiene una agenda bien diversificada que comprende lo político, lo comercial, una nutrida cooperación en defensa y seguridad y subnacional- (Colacrai, 2014). En el caso Argentina-RBV se trata de una relación de ascenso vertiginoso. Las acciones desplegadas en este vínculo mostraron cierta urgencia impulsada por la coyuntura que implicó algún grado de improvisación y menos racionalidad -los cálculos resultaron menos ajustados a las herramientas disponibles para gestionarlo-. Un dato no menor es que existen denuncias de corrupción a raíz de las cuales, las autoridades tomaron la decisión política de bajar el perfil de la relación desde 2008 lo que implicó cierto grado de estancamiento: no se incorporaron temas en la agenda, se suspendieron actividades previstas en los acuerdos, continúa pendiente el Plan de alianza estratégica y la operatividad de los mecanismos institucionales funcionó con irregularidad y discontinuidad.

Lo expuesto permite sostener que Argentina-Chile exhiben una relación ejemplar que se sustenta en bases sólidas y es más densa, compleja y profunda que el vínculo argentino-venezolano pues, a diferencia de éste, incluye la dimensión estratégica y exhibe un extenso historial político y comercial. Tanto Piñera como Fernández coincidieron en que los vínculos entre sus países demuestran continuidad y se han profundizado. No obstante, se coincide con Colacrai en "que no fueron todo lo bueno que podrían haber sido" y que, pese a ello "para uno y otro gobierno, representaron la mejor relación vecinal que pueden exhibir" (2014: 149).

En cuanto a los principios ordenadores ellos son: compromiso democrático, DDHH, Estado de derecho, pluralismo político. Desde el punto de vista discursivo se advierten coincidencias pero éstas se diluyen cuando se pone la lupa sobre su efectivo cumplimiento. Argentina-Chile respetan, de manera inequívoca, su compromiso con los principios enumerados supra mientras que en el caso Argentina-RBV se advierte una brecha entre 
discurso y praxis. Se reivindican principios centrales como ejes de la relación pero el gobierno de Chávez los incumplió en su propio sistema político (Serbín \& Serbín, 2013). Dicha administración fue denunciada por no respetar la división de poderes -injerencias del ejecutivo sobre el legislativo y judicial-, se la denunció por la existencia de presos políticos -Vivas Forero, Simonovis, Afiuni, etc-. Frente a esa situación, Argentina se mantuvo neutral durante la primera década del SXXI.

En los mecanismos institucionales se combinan semejanzas y diferencias. El marco político amplio funcionó regularmente y tal como se previó en los acuerdos entre los países vecinos. Esto facilitó el tratamiento de las cuestiones urgentes, críticas y de rutina mientras que con RBV, lo hicieron de manera aleatoria pues no se celebraron con la frecuencia establecida. También presentan similitudes en cuanto a la forma en la que se organizan y los actores que involucran -Cumbres Presidenciales, Reunión Binacional de Ministros-. Sin embargo, se advierten diferencias que pueden comprenderse tomando en consideración que Argentina y Chile tienen instituciones binacionales en el área de la seguridad y la defensa; en materia de infraestructura y, a nivel subnacional, Comités de Integración. Además, le asignan importancia a la diplomacia parlamentaria y tienen una Comisión Parlamentaria Conjunta.

El análisis de la trayectoria y las diferencias identificadas en este par de vínculos permiten interpretar que el modo en el que éstos se desarrollaron, arrojan pistas claves sobre el horizonte temporal sobre el que se proyectan. Esto es, la sostenibilidad de ambas en el mediano plazo. La planificación de los vínculos argentino-chilenos contrasta con la improvisación y el sentido de urgencia que impulsó los argentino-venezolanos. Además, se subraya que el primero partió de una situación de rivalidad intensa signada por una historia de disputas territoriales que demandó una fuerte voluntad y compromiso político para su resolución pacífica y definitiva. Desde los noventa, descansa sobre un largo y arduo proceso de cooperación con algunos episodios de tensión. Pese a ello, la historia de más tres décadas de cooperación ofrece elementos notables que permiten vislumbrar que, aún con conflictos puntuales, predominó el compromiso y la voluntad política para hallar soluciones a los problemas y para que éstos no contaminen la totalidad de la relación.

En el segundo, sería un error interpretar la ausencia de tensiones como indicador positivo a favor de esa relación pues los conflictos son intrínsecos a vínculos densos, profundos e interdependientes. El aumento de la interdependencia, inevitablemente, genera diferencias que es necesario resolver. Esto permite afirmar que el vínculo Buenos Aires-Caracas es menos denso, menos profundo y menos integral que el existente entre Buenos Aires-Santiago.

La trayectoria de la relación argentino-chilena se mantuvo constante pese al cambio de signo político de los gobiernos realizados en ambos países y a los sobresaltos que experimentó con los episodios de crisis mencionados. Mientras que la trayectoria de la relación argentino-venezolana fue en ascenso como consecuencia de la mayor convergencia de las ideas de los presidentes y porque la complementariedad existente ofrecía oportunidades para satisfacer buena parte de sus intereses y necesidades más apremiantes. El ascenso de los temas energéticos en la agenda externa y las urgencias argentinas, fueron ingredientes claves para estrechar el comercio en la materia. 


\section{Reflexiones finales}

El análisis realizado permite afirmar que las relaciones argentino-chilenas son más densas y profundas, exhiben una trayectoria de trabajo conjunto, muestran algunos avances en la búsqueda de coordinación de posiciones -en lo bilateral y en lo regionalaunque aún insuficientes. El accionar de las partes fue consecuente con la intención de constituir una alianza estratégica en sentido estricto ya que el relanzamiento de la relación en 2008 y la puesta en práctica del Tratado de Maipú son avances en esa dirección. No obstante, el proceso permanece inconcluso. La propuesta de conformar una alianza estratégica argentino-venezolana fue en sentido laxo y se ajusta a la idea de inconsecuencia retórica porque se trata de una relación menos madura, con bases endebles y donde la falta de conocimiento de las partes, constituían una desventaja para la coordinación y planificación conjunta. En el mejor de los casos se trató de una expresión de deseos que tenía exiguas chances de plasmarse en la práctica teniendo en cuenta los escasos recursos y herramientas que las partes disponían para su gestión.

En virtud de los conceptos presentados supra, se puede afirmar que pensar la política exterior como un instrumento estatal que cuenta con medios diversos tales como la capacidad de asociación con otros actores -Chile y RBV en este caso- es compatible con el concepto de alianza estratégica. En virtud de ello, se señala que estos vínculos no constituyen alianza estratégica en un sentido específico y estricto del concepto. También se reafirma que la decisión de conformar las alianzas estratégicas respondió, en los dos casos, a una decisión política de los respectivos gobiernos. En uno de ellos, partió de una base sólida y planificada mientras que en el otro se lo hizo improvisadamente y con un sentido laxo. Esto se observa en la composición de las agendas: la argentino-chilena incluye temas políticodiplomáticos, estratégicos, territoriales, comerciales, de integración cultural, científicotecnológica y subnacional mientras que la argentino-venezolana se circunscribe a lo político, diplomático y, de manera más limitada y menos transparente, en lo comercial.

Los gobiernos tomaron la decisión de avanzar hacia la alianza estratégica pero les queda camino por recorrer en materia de coordinación y planificación para hacer los cursos de acción más previsibles y confiables. Así, el proceso de construcción de la alianza estratégica permanece incompleto en ambos casos aunque existen mejores condiciones para lograrlo en sentido estricto entre Buenos Aires y Santiago.

\section{Bibliografía}

Alvear, María Soledad (2004), "La política exterior de Chile en los inicios del milenio", Diplomacia, Estrategia y Política, Brasilia, v. 1, no 1, pp. 49-67.

Aranda, Gilberto \& Riquelme, Jorge (2011), “La política exterior de Chile desde 1990. Inserción internacional y prioridad regional", Cuadernos sobre Relaciones Internacionales, Regionalismo y Desarrollo, Mérida, v. 6, no 11, pp. 11-41.

Aranda, Gilberto \& Riquelme, Jorge (2015), “¿Es posible la convergencia en la diversidad? Chile entre la Alianza del Pacífico y el Mercosur", Revista de Relaciones Internacionales, Estrategia y Seguridad, Bogotá, v. 10, no 2, pp. 155-178.

Aranda, Gilberto (2013), El proyecto Chávez (1999-2007), Santiago de Chile: Editorial Universitaria. 
Bernal-Meza, Raúl (1998), “As relações entre Argentina, Brasil, Chile e Estados Unidos: Política Exterior e Mercosul”, Revista Brasileira de Política Internacional, Brasilia, v. 41, no 1, pp. 89-107.

Briceño Monzón, Claudio (2011), "La Política Exterior de Venezuela en los Tiempos del Socialismo del Siglo XXI: Entre la autonomía y la subordinación al capitalismo global", Cuadernos sobre Relaciones Internacionales, Regionalismo y Desarrollo, Mérida, v. 6, no 12, pp. 79-103.

Briceño Ruiz, José (2010), “Venezuela y Argentina en la era de Chávez y Kirchner: ¿Coincidencia ideológica o pragmatismo?", en Bologna, Alfredo (comp.), La Política Exterior de Cristina Fernández. Apreciaciones promediando su mandato, Rosario: CERIR-UNR Editora, pp. 435-462.

Briceño Ruiz, José \& Simonoff, Alejandro (2014), Integración y Cooperación regional en América Latina: Una relectura a partir de la teoría de la autonomía, Buenos Aires: Biblos

Briones, Sebastián \& Dockendorff, Andrés (2015), “Continuidad y cambio en la política exterior chilena en el gobierno de Sebastián Piñera (2010-2014)", Estudios Internacionales, Santiago de Chile, v. 47, no 180, pp. 115-118.

Busso, Anabella (2014), "Los vaivenes de la Política Exterior Argentina re-democratizada (1983-2013)”, Estudios Internacionales, Santiago de Chile, v. 46, no 177, pp. 9-33.

Colacrai, Miryam \& Lorenzini, María Elena (2006), "La relación bilateral Argentina-Chile: el tránsito por un camino de grandes encuentros y desencuentros puntuales", en Bologna, Alfredo (comp.),. La Política Exterior del Gobierno de Kirchner, Rosario: CERIR-UNR Editora, pp. 181-218.

Colacrai, Miryam (2004), La Política Exterior Argentina hacia sus vecinos durante los 90', Documento de Trabajo no 1, Buenos Aires: CEIEG-CEMA. Disponible en: http://www.cerir.com.ar/admin/_cerir/archivos/libros/0000182/dt1_colacrai.p df. Consultado: 30/06/2013.

Colacrai, Miryam (2010), “Argentina-Chile. Las relaciones políticas y el crecimiento de un notable tejido de vínculos a escala nacional y subnacional", en Bologna, Alfredo (comp.), La Política Exterior de Cristina Fernández. Apreciaciones promediando su mandato, Rosario: CERIR-UNR Editora, pp. 321-363.

Colacrai, Miryam (2014), "La relación bilateral Argentina-Chile en clave política durante el gobierno de Piñera y los mandatos de Cristina (marzo 2010-marzo 2014): pudo haber sido mejor", en Bologna, Alfredo, La política exterior de Cristina Fernández al finalizar su mandato. Rosario: UNR-CERIR, pp. 127-152.

Collier, David (1994), "El Método Comparativo: Dos Décadas de cambios", en Sartori, Giovani \& Morlino, Leonardo (coord.), La Comparación en las Ciencias Sociales, Madrid: Alianza, pp. 51-79.

Cortés, María Julieta \& Creus, Nicolás (2009), “Argentina-Brasil. Intensidad variable en una relación estratégica inevitable", en Lechini, Gladys, Klagsbrunn, Victor \& Gonçalves, William (comps.), Argentina e Brasil. Vencendo os preconceitos. As várias arestas de uma concepção estratégica. Rio de Janeiro: Editora Revan, pp. 117-139. 
Diamint, Rut (2004), Estrategias desentonadas: Argentina y Chile, Seminario Internacional: Chile y Argentina: una mirada estratégica de cara al bicentenario. Santiago de Chile: FLACSO 23 de noviembre.

Durán, Roberto (2012), Los nexos chileno-argentinos después de 1984. En Generación de Diálogo Chile-Perú / Perú-Chile, Documento no 1: Experiencias de Paz y Buena Vecindad. Perú: Konrad Adenauer Stiftung-IDEI-IEI, pp. 33-40. Disponible en: http://www.kas.de/chile/es/publications/31702/. Consultado: 10/09/2014

Flisfich, Ángel (2011). "La Política Exterior y América del Sur". Estudios Internacionales, Santiago de Chile, $n^{\circ} 168,115-141$

Gardini, Gian Luca \& Lambert, Peter (2011), Latin American Foreign Policy: Between Pragmatism and Ideology, New York: Palgrave-MacMillan.

Gomez Saraiva, Miriam (2012), “Procesos de integración de América del Sur y el papel de Brasil: los casos de Mercosur y la Unasur", CIDOB d'Afers Internacionals, Barcelona, no 97-98, pp. 87-100.

Grieco, Joseph (1988), "Anarchy and the Limits of Cooperation: A Realist Critique of the Newest Liberal Institutionalism", International Organization, Cambridge v. 42, no 3, pp. 485-507.

Huneeus, Carlos (2007), “Argentina y Chile: el conflicto del gas, factores de política interna", Estudios Internacionales, Santiago de Chile, v. 40, no 158, pp. 179-212.

Keohane, Robert \& Nye, Joseph (1988), Poder e Interdependencia. La política mundial en transición, Buenos Aires: GEL.

Keohane, Robert (1984), After Hegemony. Cooperation and Discord in the World Political Economy, Princeton: Princeton University Press.

Kosacoff, B. (1999), Las multinacionales argentinas, una nueva ola en los noventa. Documento de trabajo $\mathrm{n}$ ㅇ 83. Buenos Aires: CEPAL.

Lechini, Gladys (2006), Argentina y África en el espejo de Brasil. ¿Política por impulsos o construcción de una política exterior?, Buenos Aires: CLACSO.

Leiras, Santiago (2015), América del Sur en los comienzos del nuevo milenio, Buenos Aires: EUDEBA.

Lorenzini, María Elena (2011), Política Exterior, Alianzas Estratégicas y Energía en América Latina. Las relaciones argentino-chilenas bajo la lupa, Rosario: Homo Sapiens Ediciones.

Maira, Luis (2006), "Las relaciones Argentina-Chile: un caso singular de combinación entre los vínculos interestatales y paradiplomacia", en Salazar, Pablo (coord.) La Paradiplomacia: las relaciones internacionales de los gobiernos locales, México: Cámara de Diputados, pp. 83-114.

Ministerio de Relaciones Exteriores de la República de Chile (2008), Declaración de Punta Arenas, 4 de diciembre.

Ministerio de Relaciones Exteriores de la República de Chile (2009), Comunicado de Prensa Conjunto III Reunión de Coordinación y Consultas sobre Asuntos Multilatera- 
les, 4 de septiembre.

Ministerio de Relaciones Exteriores, Comercio Internacional y Culto de la República Argentina (2000), Declaración Conjunta de los Señores Presidentes de la República Argentina y de la República de Chile, 19 de mayo.

Ministerio de Relaciones Exteriores, Comercio Internacional y Culto de la República Argentina (2003), Declaración de Cancilleres de la República Argentina y de la República de Venezuela, 31 de julio.

Ministerio de Relaciones Exteriores, Comercio Internacional y Culto de la República Argentina (2003), Manifiesto de Buenos Aires, 19 de agosto.

Ministerio de Relaciones Exteriores, Comercio Internacional y Culto de la República Argentina (2004), Convenio Integral de Cooperación entre la República Argentina y la República Bolivariana de Venezuela, 6 de abril.

Ministerio de Relaciones Exteriores, Comercio Internacional y Culto de la República Argentina (2005a), Comunicado Conjunto de los Presidentes de la República Argentina y de la República Bolivariana de Venezuela, 1 de febrero.

Ministerio de Relaciones Exteriores, Comercio Internacional y Culto de la República Argentina (2005b). Comunicado Conjunto de los Presidentes de la República Argentina y de la República Bolivariana de Venezuela, 11 de agosto

Ministerio del Poder Popular para la Comunicación y la Información de la República Bolivariana de Venezuela (2007), Líneas Generales del Plan de Desarrollo Económico y Social de la Nación 2007-2013, septiembre.

Miranda, Roberto (2012), "Bush-Obama y la continuidad de la sanción política a Argentina”, Intellector, Rio de Janeiro, v. 8, no 16, pp. 1-17.

Miranda, Roberto (2014), “Comercio y Política: Argentina entre las potencias y las no potencias" Latinoamérica. Revista de Estudios Latinoamericanos, México, no 59, pp. 41-67.

PROCHILE (2005), Informe Económico Chile-Argentina. Disponible en: http://www.prochile.gob.cl/wpcontent/blogs.dir/1/files_mf/1381328059Argent ina_analisis_economico_2013.pdf. Consultado: 27/08/2011.

Przeworski, Adam \& Teune, Henry (1970), The Logic of Comparative Social Inquiry, New York: Wiley.

Roark, Mariano \& Giglio, Antonela (2010), “Más allá de las ideologías. El comercio y las finanzas entre Argentina y Venezuela (2003-2008)", Íconos, Quito, no 38 pp. 8193.

Robledo, Marcos (2011), La Política Exterior de Chile 1990-2010 y la construcción social de la política internacional. Análisis preliminar y perspectivas, Working Paper ICSO-UDP. Disponible en: http://www.icso.cl/wpcontent/uploads/2011/09/Marcos-Robledo-Working-Paper-ICSO-Taller-1.pdf. Consultado: $15 / 06 / 2014$.

Rojas Aravena, Francisco (2003), "La construcción de una alianza estratégica. El caso de Chile y Argentina", en Domínguez, Jorge (comp.), Conflictos territoriales y de- 
mocracia en América Latina, Buenos Aires: Siglo XXI, pp. 85-130.

Romero, Carlos \& Mijares, Víctor (2016), “From Chávez to Maduro: Continuity and Change in Venezuelan Foreign Policy", Contexto Internacional, Río de Janeiro, v. 38, no 1 , pp. 191-227.

Ruz, María Inés (2011), "Relación Chile-Argentina”, en Ensignia, Jaime, et. al. (eds.), Política Exterior en el Chile Post Concertación: ¿quo vadis?, Santiago de Chile: Friedrich Ebert Stiftung-Fundación Chile21, pp. 147-154.

Sanjuán, Ana María (2008), “América Latina y el Bolivarianismo del siglo XXI. Alcances y desafíos de la política venezolana hacia la región”, en Lagos, Ricardo (comp.), América Latina: ¿̇Integración o Fragmentación. Buenos Aires: EDHASA, pp. 145178.

Serbín, Andrés \& Serbín Pont, Andrei (2013) "Si los derechos humanos se oponen...", Foreign Affairs Latinoamérica, México, v. 13, no 4, pp. 39-44.

Serbín, Andrés \& Serbin Pont, Andrei (2014), “Quince años de política exterior bolivariana ¿entre el soft- balancing y la militarización?", Pensamiento Propio, Buenos Aires, no 39 pp. 287-326.

Serbín, Andrés (2010), Chávez, Venezuela y la reconfiguración política de América Latina y el Caribe, Buenos Aires: Siglo XXI-Plataforma Democrática.

Simonoff, Alejandro (2010), La Argentina y el Mundo frente al Bicentenario de la Revolución de Mayo, La Plata: Universidad Nacional de La Plata.

Simonoff, Alejandro (2013), “Una visión estructural de la Política Exterior Argentina y el rol de Chile desde el proceso de democratización de 1983", Sí Somos Americanos, Santiago de Chile, vol. 13, $n^{\circ} 1$, pp. 15-38

Simonoff, Alejandro (2009), "Regularidades de la Política Exterior de Néstor Kirchner", Confines de Relaciones Internacionales y Ciencia Política, Monterrey, vol. 5, $\mathrm{n}^{\circ}$ 10, pp. 71-86

Stinchcombe, Arthur (1978), Theoretical Methods in Social History, Nueva York: Academic Press.

Tratado de Maipú de Integración y Cooperación entre la República Argentina y la República de Chile (2009), 30 de octubre, Maipú.

Valdés, Juan Gabriel (1999), La Política Exterior de Chile y el Proceso de Integración con Argentina, Colección Cuadernos, Serie nํ 1, Conferencias nㅇ 30, Buenos Aires: CARI.

Wendt, Alexander (1999), Social Theory of International Politics. New York: Cambridge University Press. 